García-Fernández, P.; Guodemar-Pérez, J.; Ruiz-López, M.; Rodríguez-López, E.S.; GarcíaHeras, A.; Hervás-Pérez, J.P. (2019) Epidemiology of Injuries in Professional and Amateur Spanish Paddle Players. Revista Internacional de Medicina y Ciencias de la Actividad Física y el Deporte vol. 19

$\mathrm{pp}$. 641-654

Http://cdeporte.rediris.es/revista/revista76/artepidemiologia1090.htm

DOI: $10.15366 /$ rimcafd2019.76.006

\title{
ORIGINAL
}

\section{EPIDEMIOLOGÍA LESIONAL EN JUGADORES ESPAÑOLES DE PADEL PROFESIONALES Y AMATEUR}

\section{EPIDEMIOLOGY OF INJURIES IN PROFESSIONAL AND AMATEUR SPANISH PADDLE PLAYERS}

\author{
García-Fernández, P.'; Guodemar-Pérez, J.2; Ruiz-López, M.³; Rodríguez-López, \\ E.S. ${ }^{2}$; García-Heras, A. ${ }^{4}$; Hervás-Pérez, J.P. ${ }^{2}$ \\ ${ }^{1}$ Departamento de Radiología, Rehabilitación y Fisioterapia, Facultad de Medicina, Universidad \\ Complutense de Madrid, Madrid (España) pablga25@ucm.es \\ ${ }^{2}$ Departamento de Fisioterapia, Facultad de Salud, Universidad Camilo José Cela. Villanueva de \\ la Cañada, Madrid (España) jguodemar@ucjc.edu, esrodriguez@ucjc.edu, jphervas@ucjc.edu \\ ${ }^{3}$ Departamento de Enfermería, Facultad de Salud, Camilo José Cela. Villanueva de la Cañada, \\ Madrid (España) mrlopez@ucic.edu \\ 4 Departamento de Fisioterapia, Facultad de Ciencias de la Salud, Universidad Alfonso X, \\ Villanueva de la Cañada, Madrid (España) aranka 76@hotmail.com
}

Código UNESCO / UNESCO code: 3212 Salud pública / Public Health Clasificación del Consejo de Europa / Council of Europe Classification: 11 Medicina del Deporte / Sport Medicine

Recibido 23 de enero de 2018 Received January 23, 2018

Aceptado 12 de febrero de 2018 Accepted February 12, 2018

\section{RESUMEN}

Se realizó un estudio epidemiológico descriptivo, observacional y retrospectivo, analizando la incidencia y las características de las lesiones sufridas por jugadores de pádel españoles profesionales y no profesionales. Se registraron las lesiones sufridas por 478 jugadores entre el 1 de enero y el 31 de diciembre de 2016. Se registraron datos sobre el número de lesiones, lesiones más frecuentes, distribución, tipo, ubicación, el momento en que ocurrieron y la causa de las lesiones que afectan el sistema locomotor. La tasa de lesiones en esta población fue de 2,75 lesiones por 1000 horas de exposición al riesgo. Las lesiones leves y aquellas con mecanismos de lesión intrínseca fueron las más frecuentes, y tuvieron lugar el final de la práctica deportiva. La extremidad inferior fue el área corporal más lesionada, y el tejido más lesionado fue el sistema musculo-tendinoso. A su vez, la epicondilitis fue la patología más común. 
PALABRAS CLAVE: pádel; epidemiología; lesión; extremidades inferiores; músculos

\begin{abstract}
An epidemiological study was conducted using retrospective, observational and descriptive methodologies. We sought to analyse the incidence and features of injuries sustained by professional and non-professional Spanish Padel players. In total, 478 injuries were registered in 2016 from January $1^{\text {st }}$ to December $31^{\text {st }}$. We collected data on the number of injuries, the most frequent injuries, their distribution, type, location, the moment at which these occurred and the cause of the injuries affecting the locomotor system. We found that the injury rate in this population was 2.75 injuries per 1000 hours of risk exposure. The most frequent injuries were those which were mild or with mechanisms of intrinsic injury, and most occurred towrdas the end any given game or practice. The lower limb was the most frequently injured body area, and the most injured tissue was the muscle-tendinous system. In turn, epicondylitis was the most common pathology.
\end{abstract}

KEYWORDS: Padel; epidemiology; injury; lower extremity; muscles.

\title{
INTRODUCCIÓN
}

El pádel es un híbrido entre los deportes de pared y los de red que se juega en parejas en una pista totalmente cerrada por fondos de longitud interior de 10 metros y por laterales de longitud interior de 20 metros separados por una red dispuesta a una altura de 0,88 metros. La Federación Internacional de Pádel es el organismo que rige este deporte y vela por el cumplimiento y modificación de un sistema de reglamentación de 17 normas (Federación Internacional de Pádel, 2016; Courel-Ibáñez et al., 2017).

A diferencia de otros deportes como el fútbol, donde la International Federation of Association Football (FIFA) elaboró de forma consensuada una definición de lesión (Fuller et al., 2006a; Fuller et al., 2006b), en el pádel no hay una definición de consenso sobre qué es considerado lesión, algo que dificulta el llevar a término estudios epidemiológicos y que además puedan ser comparados con otros deportes.

Son escasos los estudios que, sobre las lesiones durante la práctica del pádel, se han llevado a cabo, y éstos, sitúan el índice de incidencia personal, número de jugadores lesionados por cada 100 deportistas, en una horquilla que desde el $49,8 \%$ al $86,7 \%$. (Castillo-Lozano y Casuso -Holgado, 2015; Castillo-Lozano y Alvero-Cruz, 2016).

Los miembros inferiores se han señalado como la ubicación general más frecuente de las lesiones en el pádel, si atendemos a la ubicación precisa, el complejo articular del codo es el más afectado, siendo el tejido de asiento más 
habitual de las lesiones el musculotendinoso (Castillo-Lozano y CasusoHolgado, 2015; García González y otros, 2015; Castillo-Lozano y Alvero-Cruz, 2016).

Son escasos los estudios realizados que analicen las lesiones en el pádel y al tratarse de un deporte que cuenta con millones de practicantes (Gil Solés, 2014), se plantea como objetivo del presente estudio el analizar a los jugadores profesionales y no profesionales en la Comunidad de Madrid para describir y evaluar los datos del número de lesiones, las lesiones más frecuentes, la distribución, tipo, localización (tejidos y localización anatómica), momento en que se producen y causa de las lesiones del aparato locomotor.

\section{MATERIAL Y MÉTODOS}

\section{Diseño del estudio}

Estudio epidemiológico observacional, descriptivo, retrospectivo de tipo transversal.

\section{Métodos}

Se realizó un muestreo por conglomerados en una etapa, considerando todos los clubes pertenecientes a la Federación Madrileña de Pádel.

Se decidió incluir en el estudio a todos los jugadores de pádel federados con edades comprendidas entre los 14 y 65 años, de cualquier nivel de juego, de ambos sexos, hubieran tenido o no lesión en los últimos doce meses previos a la realización del estudio, para poder determinar la incidencia y prevalencia de lesiones durante la práctica de pádel.

Sobre un total de 130 clubes, la muestra estuvo compuesta por los 5 clubes con mayor número de licencias que acepatron participar en el estudio. Después de informar a los 576 jugadores federados pertenecientes a los clubes seleccionados de la realización y los objetivos del estudio de investigación, 514 (83.5\%) jugadores se ofrecieron para participar. De estos, 478 (77.7\%) cumplían los criterios de inclusión, resultando esta muestra significativa, al $95 \%$ de nivel de confianza y con un intervalo de confianza de 1.96 sigmas, para la población de estudio. Un $61 \%$ fueron jugadores aficionados, un $26 \%$ semiprofesionales, un $4,5 \%$ profesionales y un $8,5 \%$ entrenadores.

Todos los participantes firmaron un consentimiento informado antes de la recogida de datos y el estudio se realizó de acuerdo con los principios de la declaración de Helsinki para la investigación con seres humanos (WMA, 2013).

\section{Procedimiento}

Se recogieron todas las lesiones producidas desde el 1 de enero al 31 de diciembre de 2016. Las encuestas se realizaron, siempre por la mismo personal 
investigador, durante el periodo comprendido entre el 1 de febrero al 30 de marzo de 2017.

Se incluyeron en el estudio todas las lesiones producidas durante el entrenamiento o competición durante la práctica de pádel. La totalidad de los entrenamientos y partidos se desarrollaron en césped artificial o moqueta.

Se consideró lesión toda aquella incidencia que precisó tratamiento sanitario de cualquier tipo, o causado baja en entrenamiento o competición (Ekstrand et al., 2004). Los jugadores con una o varias lesiones debían haber conseguido una recuperación completa de estas lesiones en estudio para un correcto análisis de sus consecuencias. Se incluyeron en el estudio las lesiones de nueva aparición durante el periodo a investigar, excluyéndose recidivas de lesiones previas, lesiones crónicas, así como cualquier lesión no relacionada con la práctica de este deporte.

\section{Instrumentos}

El presente estudio utilizó un formulario de recopilación de datos siguiendo las últimas recomendaciones para la realización de estudios en el tenis (Pluim et al., 2009). En dicho cuestionario se recopilaron datos personales, antropométricos, datos sobre la práctica deportiva de Pádel y datos específicos sobre las lesiones sufridas.

Las lesiones se clasificaron de acuerdo con su ubicación anatómica en áreas principales y categorías equivalentes al sistema OSICS (Sistema de Clasificación de Lesiones Deportivas Orchard), (Schoffl et al., 2011). Para clasificar las lesiones según el tipo se utilizó el citado sistema OSICS (versión 10) que incluye un total de 1.626 posibles diagnósticos (Rae y Orchard, 2007).

\section{Análisis estadístico}

Se utilizó el paquete estadístico SPSS versión 21,0 para Windows para el análisis estadístico, considerando $p<0,05$.

Se calcularon los coeficientes de correlación de Pearson y se interpretaron sobre la base de las variables cuantitativas, para analizar las correlaciones, así como los coeficientes de correlación de Spearman cuando se utilizaron variables categóricas. Se calcularon las pruebas de contraste de Chi-cuadrado para analizar la asociación entre variables cualitativas con respecto a lesiones y atletas.

Se utilizó la prueba de Kolmogorov-Smirnov para analizar si las variables cuantitativas se distribuían normalmente, con el fin de comparar los medios mediante la prueba t de Student en el caso de dos medias o el ANOVA en el caso de tres o más medias. Cuando no se encontró normalidad en la distribución de los datos, se utilizó la prueba no paramétrica de Wilcoxon para comparar dos medias y se utilizó la prueba de Kruskal-Wallis para comparar dos o más medias (Pérez, 2009). 


\section{RESULTADOS}

\section{Población de estudio}

Se obtuvieron resultados de un total de 478 jugadores de pádel federados, pertenecientes a diferentes clubes de la Comunidad de Madrid, España. De estos, $332(69.5 \%)$ eran hombres y 146 (30.5\%) mujeres.

\section{Incidencia lesional}

Se registraron 515 lesiones en total. La incidencia lesional o el número de lesiones por 1000 horas de exposición al riesgo fue de 2,75 lesiones por 1000 horas de práctica deportiva y 8,44 lesiones por 1000 partidos, por lo que la tasa de lesiones personales o el número de deportistas lesionados por cada deportista fue de $65,6 \%$. La tasa de lesiones o el número de lesiones por cada 100 jugadores fue de 108 lesiones. El número de lesiones aumentó significativamente con la edad $(p=0,045)$ y con el aumento del IMC $(p=0,009)$.

\section{Región corporal afectada}

Las lesiones que afectaron la extremidad inferior fueron las más frecuentes, con 211 lesiones registradas $(41,0 \%)$, seguidas de las de la extremidad superior con 183 lesiones registradas (35,5\%) (Figura 1 ).

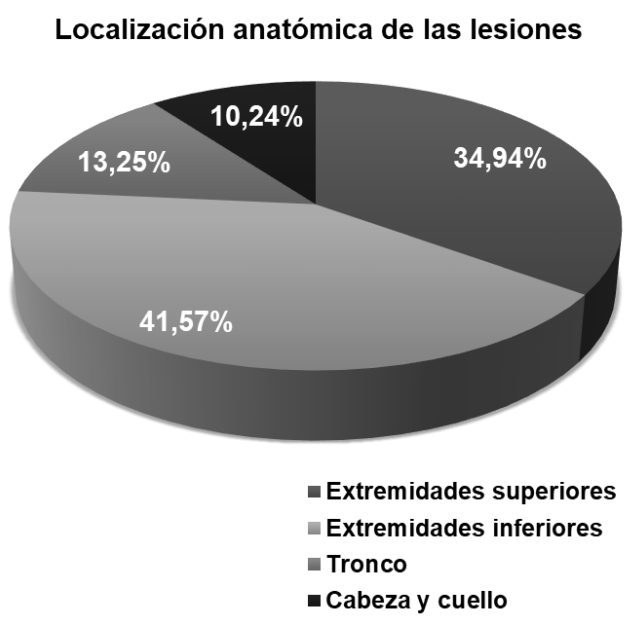

Figura 1. Localización anatómica de las lesiones

En cuanto a la ubicación exacta, el área más afectada fue el codo, con 105 lesiones registradas $(20,48 \%)$, seguidas de pie y rodilla, con $59(11,45 \%)$ y 56 $(10,84 \%)$ lesiones, respectivamente (Tabla 1$)$. 


$\begin{array}{lcc}\text { Localización anatómica de las } & \text { Frecuencia } & \text { Porcentaje } \\ \text { lesiones } & 105 & 20,48 \% \\ \text { Codo } & 59 & 11,45 \% \\ \text { Pie } & 56 & 10,84 \% \\ \text { Rodilla } & 47 & 9,04 \% \\ \text { Columna lumbar } & 44 & 8,43 \% \\ \text { Hombro } & 44 & 8,43 \% \\ \text { Pierna / Región sural } & 44 & 8,43 \% \\ \text { Cuello / Columna Cervical } & 40 & 7,83 \% \\ \text { Tobillo } & 25 & 4,82 \% \\ \text { Muñeca } & 12 & 2,42 \% \\ \text { Columna Dorsal } & 9 & 1,81 \% \\ \text { Región Femoral / Muslo } & 9 & 1,81 \% \\ \text { Pelvis / Sacro / Glúteo } & 9 & 1,81 \% \\ \text { Cabeza / Cara } & 6 & 1,20 \% \\ \text { Brazo } & 3 & 0,60 \% \\ \text { Antebrazo } & 3 & 0,60 \% \\ \text { Dedos } & \mathbf{1 0 0 , 0 0 \%}\end{array}$

Tabla 1. Región del cuerpo afectada

Tejido y lesión más frecuente

Las lesiones tendinosas fueron las más frecuentes, $40,4 \%$, seguidas de lesiones musculares, que representaron el 30,7\%. En tercer lugar, las lesiones ligamentosas totalizaron el $17,5 \%$ de las lesiones totales (Tabla 2).

$\begin{array}{lcc}\text { Tejido } & \text { Frecuencia } & \text { Porcentaje } \\ \text { Articulaciones y ligamentos } & 90 & 17,5 \% \\ \text { Muscular } & 159 & 30,7 \% \\ \text { Tendinoso } & 208 & 40,4 \% \\ \text { Óseo } & 6 & 1,2 \% \\ \text { Piel } & 0 & 0,0 \% \\ \text { Otros } & 52 & 10,2 \% \\ \text { Total } & 515 & 100,0 \%\end{array}$

Tabla 2. Tejido involucrado

En cuanto al diagnóstico preciso, la epicondilitis fue la lesión más frecuente: representó el $20,5 \%$ del total, seguida de la fascitis plantar y las contracturas cervicales que, en ambos casos, representaron $8,4 \%$ y de los esguinces que afectaron al ligamento lateral externo del tobillo que representaron un $7,8 \%$ (Figura 2). Se ha podido objetivar una dependencia estadísticamente significativa entre el nivel de juego y el tipo de lesión $(p=0,032)$, los jugadores aficionados sufren tendinopatías y fascitis plantar con mayor frecuencia, 
mientras que los profesionales presentaron un mayor número de sobrecargas musculares.

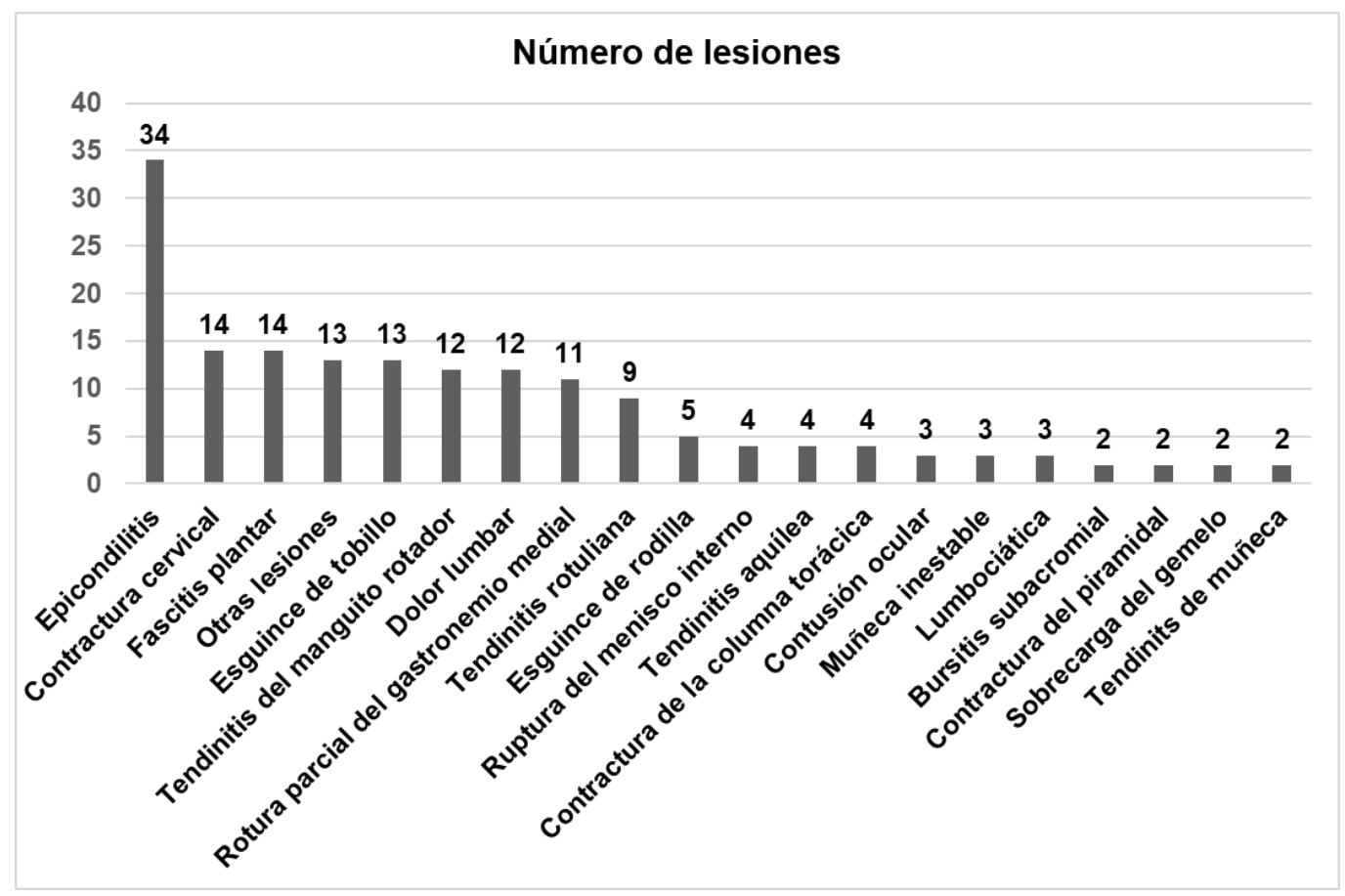

Figura 2. Número de lesiones por tipo

Motivo de la lesión y severidad

El motivo lesional que se registró con más frecuencia fue el de no contacto, representando un $68,0 \%$ de total, siendo la sobrecarga muscular con un $42,8 \%$ el motivo más frecuente entre las de este tipo, observándose una fuerte correlación entre este motivo lesional y las lesiones que afectan al hombro y al codo $(p=0,000)$. Las de carácter extrínseco fueron un $32,0 \%$ destacando entre estas el mal estado de las instalaciones, $11,0 \%$, o el deterioro y/o mal estado del material deportivo con un porcentaje del $6,4 \%$.

La severidad de las lesiones, siguiendo la propuesta de consenso para estudios epidemiológicos en el tenis de Pluim et al. (2009), se clasificó en base a los días de baja que motivaban. Lesiones de carácter mínimo (1-3 días), leve (4-7 días), moderado (8-28 días), grave (> 28 días a 6 meses). Las lesiones leves fueron las más frecuentes con un $30,1 \%$, presentando asociación estadísticamente significativa con las lesiones musculo-tendinosas y que afectaron a los miembros superiores $(p=0,041)$. Las lesiones moderadas supusieron el $28,3 \%$ y en este caso presentaron significación estadística con las lesiones ligamentosas y las que se asentaron en los miembros inferiores $(p=0,047)$. Las recidivas fueron del $25,0 \%$, presentando una asociación estadísticamente significativa con las lesiones que afectaron al codo $(p=0,018)$.

En el pádel, el 40,0\% de las lesiones no precisan de asistencia sanitaria ni motivan baja laboral. En cuanto a las que sí lo precisaron, el 50,0\% necesitó de un máximo de 10 sesiones de fisioterapia. Existe una asociación 
estadísticamente significativa entre el número de sesiones, la entidad de la lesión y las secuelas de las mismas, objetivándose que las lesiones de mayor severidad reciben más atención fisioterápica y presentan menos secuelas.

El $57,2 \%$ de las lesiones producidas durante la práctica del pádel no produjo ninguna secuela. Entre las que sí lo hicieron y como secuela dejaron dolor, este presento una asociación estadísticamente significativa con las lesiones tendinosas que afectaron a codo y hombro $(p=0,000)$.

\section{Equipamiento y hábitos deportivos}

En relación al material empleado por los jugadores, la pala más utilizada es la de tipo lágrima, $35,0 \%$, el $35,7 \%$ de los encuestados utilizan palas con un peso inferior a los 370 gramos, siendo ésta en el $55,0 \%$ de los casos, de superficie blanda. La utilización de un grip fino y 1 overgrip es lo más frecuente.

En lo que al calzado se refiere, el $97,4 \%$ utiliza calzado de caña baja específico de pádel, siendo la suela tipo espiga la más empleada, $70,0 \%$. Además, los jugadores de pádel no utilizan una sola superficie de juego, siendo el $64,0 \%$ los que alternan diferentes tipos de pista.

El $61,0 \%$ de los jugadores de pádel lo hace de forma recreativa y dedica fundamentalmente el tiempo de práctica al entrenamiento con clase, dedicando de forma semanal 8,15 horas de media y disputa 2,9 partidos por semana.

\section{DISCUSIÓN}

Los hallazgos de nuestro estudio han revelado una incidencia lesional de 2,75 lesiones por cada 1000 horas de práctica deportiva de pádel con 8,44 lesiones por cada 1000 partidos. Estudios descriptivos epidemiológicos en tenis, con diseño de estudio y definición de lesión comparables al nuestro, informan de una incidencia lesional similar a la nuestra: 2,3 (Winge et al., 1989) y 3,0 (Jayanthi et al., 2005). El índice lesional personal o cantidad de jugadores lesionados por cada 100 jugadores (dado que 313 jugadores resultaron lesionados de 478 jugadores en total), fue de $65,6 \%$. En otros estudios epidemiológicos de pádel informan de un índice lesional personal de entre $49,9 \%$ y $86,70 \%$, por lo tanto, nuestro estudio está en el rango medio de esa horquilla (Castillo-Lozano y Casuso-Holgado, 2015; García González et al., 2015; Castillo-Lozano y AlveroCruz, 2016).

En cuanto a la ubicación anatómica de las lesiones, la extremidad inferior fue la más afectada en nuestro estudio con un $41,0 \%$, seguida de la extremidad superior $(35,5 \%)$ y el tronco $(13,0 \%)$. Se han observado resultados similares con respecto a la ubicación anatómica general en el estudio de García González et al., (2015) y en el de Castillo-Lozano y Alvero-Cruz (2016). En el tenis son varios los estudios que informan de mayor incidencia en las extremidades inferiores (Steinbruk, 1999; Kuhne et al., 2004; Jayanthi et al., 2005), también es el caso del bádminton y squash (Caine et al., 1996; Fortington et al., 2015). Este hecho puede ser debido a las características propias de este tipo de juegos, en donde 
se producen cambios de dirección bruscos, aceleraciones y deceleraciones de gran intensidad, saltos con recepciones inestables, etc. Todo ello conlleva una alta solicitación tanto a nivel articular como muscular motivando así un elevado número de lesiones en las extremidades inferiores.

El codo fue la zona de asiento más frecuente con un $20,5 \%$ de todas las lesiones, seguido del pie $(11,4 \%)$, la rodilla $(10,8 \%)$ y la región lumbar en el $9,0 \%$. Otros estudios de pádel describen resultados idénticos, identificando el codo como el área principal de lesión (Castillo-Lozano y Casuso-Holgado, 2017; CastilloLozano y Casuso-Holgado, 2015; Castillo-Lozano y Alvero-Cruz, 2016). El alto porcentaje de lesiones que tiene el codo como zona de asiento lesional, podemos imputarlo a la alta solicitación del complejo articular del codo, las continuas contracciones excéntricas que se producen tras el golpeo para frenar el movimiento, y que la pala de pádel no presenta un cordaje en el que se pueda regular la tensión, lo que en nuestra opinión contribuiría de forma decisiva en la disminución de la implicación de la zona al realizar el gesto técnico del golpeo.

Las lesiones musculo-tendinosas halladas han sido las más frecuentes, seguidas de las lesiones articulares y ligamentosas. Estos mismos resultados han sido encontrados en el estudio epidemiológico en pádel publicado por García González et al., (2015). En cuanto al tipo preciso de las lesiones, predominó la tendinopatía con 208 lesiones (38,5\%), fundamentalmente en forma de epicondilitis $(20,5 \%)$ y tendinitis del manguito de los rotadores $(7,8 \%)$, datos que concuerdan con el estudio de García González et al., (2015) y con otros deportes de raqueta (Caine et al., 1996; Jayanthi et al., 2005). Posiblemente estos datos se deban a una mala técnica en la ejecución del golpe de revés, a la ausencia de acciones preventivas o la mala elección de la pala. Por ello creemos que el dedicar tiempo al trabajo técnico en la ejecución del gesto deportivo, sobre todo en las etapas iniciales y los niveles de formación, junto con sesiones de fortalecimiento muscular general en donde se dé importancia a la contracción excéntrica específica y controlada de la musculatura más implicada en el golpeo, contribuiría de forma decisiva en la reducción de este tipo de lesiones. Sin olvidar que la elección de la pala se realiza en muchas ocasiones según criterios estéticos o publicitarios y no de acuerdo a la morfología o necesidades del jugador siendo esta una causa frecuente de lesión.

Las lesiones de carácter intrínseco fueron las más frecuentes, hecho contrastado en el estudio de García González et al., (2015). Se ha podido constatar que las lesiones por no contacto afectan con más frecuencia a los miembros superiores y las de carácter extrínseco tienen atracción por los miembros inferiores, algo que también se observó en la tipología las lesiones, las que afectaban a meniscos y ligamentos tienen un origen extrínseco y las que afectaron a músculos y tendones carácter intrínseco.

El 25,0\% de las lesiones en nuestro estudio fueron recidivantes. Estos hallazgos son similares a otros estudios de pádel, como el de Navarro et al., (2013) en el que el $23,5 \%$ de las lesiones fueron recidivas de una lesión que ocurrió antes del período de estudio, mientras que, durante el período de estudio, el $24,5 \%$ de las lesiones recidivó. 
En el $42,0 \%$ de los casos, las lesiones ocurrieron durante la fase final de la actividad deportiva. Este hallazgo está respaldado por un estudio epidemiológico en el que participaron 400 jugadores de pádel que informa de datos similares, y que definieron esta fase como la más lesiva (García González et al., 2015). Además, se evidenció una fuerte asociación entre las lesiones que afectan las extremidades superiores y la etapa final de los encuentros seguramente debido a la sobresolicitación y falta de preparación física y/o técnica, mientras que las lesiones de las extremidades inferiores se produjeron principalmente al comienzo de los partidos creemos que debido a un inadecuado calentamiento del tren inferior.

Con respecto a los hábitos deportivos de los participantes en el estudio, la alternancia de la pista cubierta con juego al aire libre fue lo más frecuente y los jugadores que alternaron la posición de revés y de derecha lo más común en nuestro estudio. Comparando estos datos con otros estudios se observan diferentes combinaciones de tipo de pista y posición, por lo que no es posible inferir una clara tendencia respecto de estas variables (Castillo-Lozano y Casuso-Holgado, 2015; García González et al., 2015; Castillo -Lozano y AlveroCruz, 2016).

El 62,0\% de los jugadores tenían más de seis años de experiencia practicando pádel, porcentaje similar al de otro estudio realizado en 130 jugadores españoles de pádel (Castillo-Lozano y Casuso-Holgado, 2017), dedicando un promedio de 8,15 horas por semana a la práctica de pádel, jugando 2,9 partidos semanales. Ninguno de estos factores ha demostrado ser relevante en términos de un aumento significativo en el número de lesiones ni en el tipo o ubicación de las mismas. No se han encontrado estudios de pádel que informen o relacionen estas variables. En el caso del tenis, el aumento del tiempo de práctica se asoció con una mayor incidencia de nuevos casos de epicondilitis en jugadores de tenis recreacionales que jugaban más de dos horas al día, en comparación con aquellos que jugaban menos de dos horas al día (Gruchow y Pelletier, 1979). Sin embargo, en el estudio de Jayanthi et al., (2005), al analizar la incidencia de las lesiones sufridas por los jugadores de tenis no informó de ninguna diferencia entre los jugadores que jugaron menos de cuatro horas a la semana, de cuatro a seis horas o más de seis horas por semana. En consecuencia, la posible relación entre tiempos de juego mayores y un aumento de la incidencia lesional en deportes de raqueta no está clara.

La raqueta más común utilizada fue de tipo lágrima, con un peso menor de 370 gramos y en el $55,0 \%$ de los casos, con una superficie blanda. Observamos una tendencia a la asociación entre las lesiones que afectan el miembro superior y las raquetas redondas, que pesan entre 371 y 380 gramos. El uso de un agarre fino y un sobregrip es lo más común, aunque este no es un factor significativo en la aparición de lesiones, su ubicación o la tipología de las mismas. No se han podido encontrar otros estudios de pádel que analizaran el ancho del agarre y su relación entre las ubicaciones y los tipos de lesiones. Nirschl (1992) especuló que sería necesario un agarre más cerrado o duro si el grip es pequeño, aumentando la actividad muscular o necesitaría menos fuerza con un grip más grueso, según este autor el uso de un tamaño del grip adecuado, disminuiría el esfuerzo necesario para sostener la raqueta, aumentando la eficiencia de los 
músculos del antebrazo y mano, y disminuyendo la posibilidad de lesiones por sobreuso de las EESS. Varios estudios retrospectivos con tenistas recreativos mostraron una asociación entre la incidencia de epicondilitis y el uso de un grosor de puño inadecuado, bien demasiado pequeño o demasiado grande (Gruchow y Pelletier, 1979; Hang y Peng, 1984). En otro estudio más reciente en el que se evaluó la fuerza empleada por los músculos extensores de la muñeca en jugadores de tenis intermedios y avanzados, utilizando tres anchos de agarre diferentes, concluyeron que el tamaño de agarre reduce los factores de riesgo mecánicos que producen lesiones por sobrecarga en las extremidades superiores (Rossi et al., 2014). Sin embargo, en un estudio de laboratorio controlado con 16 jugadores de tenis asintomáticos, no se encontraron diferencias estadísticamente significativas en la actividad electromiográfica de los músculos epicondileos entre los jugadores que usaron un agarre que era más pequeño, mayor o igual que el recomendado por Nirschl (1992) en el golpe de revés (Hatch et al., 2006). Es posible que el grosor de agarre sea un factor de riesgo mecánico para la aparición de lesiones por uso excesivo en la extremidad superior.

El 97,4\% de los jugadores utiliza calzado de caña baja específico de pádel, siendo la suela tipo espiga la más empleada, $70,0 \%$. El utilizar suela de tipo omni se ha demostrado un factor que predispone de forma significativa a sufrir lesiones en los miembros inferiores, presentando rechazo por esta ubicación en las lesiones los otros tipos de suela. En la localización anatómica precisa y en la tipología no hemos encontrados correlaciones significativas. No se han encontrado en la literatura estudios de pádel que analicen la relación entre el calzado y la localización y tipología lesional.

Las lesiones en el pádel están poco influenciadas por el nivel de juego, solamente hemos hallado diferencias estadísticamente significativas entre jugadores aficionados y profesionales en cuanto a la ubicación general de las lesiones, siendo las lesiones de cabeza y cuello las más frecuentes en el grupo de profesionales, y en cuanto a la tipología, las lesiones musculares, concretamente las contracturas, son más frecuentes en el grupo de profesionales. Posiblemente esto sea debido al reducido número de jugadores profesionales en relación al abundante grupo de jugadores federados de nivel aficionado.

\section{Limitaciones}

En el presente estudio el registrar los datos mediante un cuestionario sobre las lesiones del año anterior puede suponer un sesgo dado que en alguna ocasión se puede perder información relevante, y en ocasiones, esta información puede ser influenciada por aspectos subjetivos como el dolor percibido por el jugador.

\section{CONCLUSIONES}

La lesión de carácter leve y mecanismo intrínseco es la más frecuente en el pádel, presentando una incidencia lesional similar a la de otros deportes de raqueta. 
El tejido musculo-tendinoso es el que sufre mayor número de lesiones, siendo la patología más común la epicondilitis. El miembro inferior es el más frecuentemente afectado y la ubicación específica más habitual es el codo.

Los jugadores de pádel disputan una media de 2,9 partidos semanales jugando en diferentes tipos de superficie y utilizando de forma más frecuente la pala con forma de lágrima y superficie blanda.

\section{REFERENCIAS BIBLIOGRAFICAS}

Bishop D, Bonetti D, Spencer M. The effect of an intermittent, high-intensity warm-up on supramaximal kayak ergometer performance. J Sports Sci, 2003; 21(1), 13-20. https://doi.org/10.1080/0264041031000070912

Caine CG, Caine DJ, Lindner KJ. The Epidemiologic Approach to Sports Injuries. In C. G. Caine, DJ. Caine KJ. Lindner (Eds.), Epidemiology of sports injuries, 1996; pp. 1-13. Champaign, Illinois: Humans Kinetics. https://trove.nla.gov.au/version/45620150

Castillo-Lozano R, Alvero-Cruz JR. Estudio epidemiológico de las principales lesiones músculo-esqueléticas en jugadores de pádel. Innovación e investigación en pádel, 2016; pp. 21-38. In J. Courel, B. J. SánchezAlcaraz \& J. Cañas (Eds.), Sevilla: Wanceulen. ISBN 9788499933887

Castillo-Lozano R, Casuso-Holgado MJ. Incidence ofmusculoskeletal sport injuries in a sample of male and female recreational paddle-tennis players. Sports Med Phys Fitness, 2017;57(6):816-821. https://doi.org/10.23736/S0022-4707.16.06240-X

Castillo-Lozano R, Casuso-Holgado MJ. A comparison muskuloskeletal injuries among junior and senior paddle-tennis players. Science \& Sports, 2015; 30(5), 268-274. https://doi.org/10.1016/j.scispo.2015.03.005

Courel-Ibáñez J, Sánchez-Alcaraz Martínez BJ, Cañas J. "Game Performance and Length of Rally in Professional Padel Players" Journal of Human Kinetics, 2017; 55(1): 161-169. https://doi.org/10.1515/hukin-2016-0045

Ekstrand J, Waldén M, Hägglund $M$. A congested football calendar and the wellbeing of players: Correlation between match exposure of European footballers before the World Cup 2002 and their injuries and performances during that World Cup. Br J Sports Med. 2004;38:493-

7. https://doi.org/10.1136/bjsm.2003.009134

F.I.P. Federaciones. Miembros asociados, 2016; Retrieved 25/01/2016, from http://www.padelfip.com/\#!federaciones/c225f

F.I.P. Reglamento de juego de pádel, 2008; Retrieved 26/01/2016, from http://www.padelfederacion.es/refs/docs/REGLAMENTO FIP.pdf

Fortington LV, Donaldson A, Lathlean T, Young WB, Gabbe BJ, Lloyd D, Finch CF, When 'just doing it' is not enough: Assessing the fidelity of player performance of an injury prevention exercise program. Journal of Science and Medicine in Sport, 2015; 18(3), 272-277. https://doi.org/10.1016/j.jsams.2014.05.001

Fuller CW, Ekstrand J, Junge A, Andersen TE, Bahr R, Dvorak J, Meeuwisse $\mathrm{WH}$. Consensus statement on injury definitions and data collection 
procedures in studies of football (soccer) injuries. Br J Sports Med, 2006; 40(3), 193-201. https://doi.org/10.1136/bjsm.2005.025270

Fuller CW, Ekstrand J, Junge A, Andersen TE, Bahr R, Dvorak J, Meeuwisse WH. Consensus statement on injury definitions and data collection procedures in studies of football (soccer) injuries. Clin J Sport Med, 2006; 16(2), 97-106. https://doi.org/10.1097/00042752-200603000-00003

García González C, Albaladejo Vicente R, Villanueva Orbáiz R, Navarro Cabello E. Deporte de ocio en España: epidemiología de las lesiones y sus consecuencias (Epidemiological Study of Sports Injuries and their Consequences in Recreational Sport in Spain). Apunts. Educación Física y Deportes, 2015; 119(3), 62-70. https://dx.doi.org/10.5672/apunts.20140983.es.(2015/1).119.03

Gil Solés D. Historia del pádel, 2014; Retrieved 15/04/15, from http://padelads.com/historia-del-padel/

Gruchow HW, Pelletier D. An epidemiologic study of tennis elbow. Incidence, recurrence, and effectiveness of prevention strategies. Am J Sports Med, 1979; 7(4), 234-238. https://doi.org/10.1177/036354657900700405

Hang YS, Peng SM. An epidemiologic study of upper extremity injury in tennis players with a particular reference to tennis elbow. Taiwan Yi Xue Hui Za Zhi, 1984; 83(3), 307-316. PMID:6588142

Hatch GF, Pink MM, Mohr KJ, Sethi PM, Jobe FW. The effect of tennis racket grip size on forearm muscle firing patterns. Am J Sports Med, 2006; 34(12), 1977-1983. https://doi.org/10.1177/0363546506290185

Jayanthi N, Sallay P, Hunker P, Przybylski M. Skill-level related injuries in recreational competition tennis players. Med Sci Tennis, 2005; 10(1), 1215.

Kuhne CA, Zettl RP, Nast-Kolb D. Injuries- and frequency of complaints in competitive tennis- and leisure sports. Sportverletz Sportschaden, 2004; 18(2), 85-89. https://doi.org/10.1055/s-2004-813049

Navarro, E., Albaladejo, R., Villanueva, R., García, C., Majón, R., \& Hernández, J. V. (2013). Estudio epidemiológico de las lesiones en el deporte de ocio: Parte II: pádel. Madrid: Fundación MAPFRE.

Nirschl, RP. Elbow tendinosis/tennis elbow. Clin Sports Med, 1992; 11(4), 851870. PMID: 1423702

Pérez C. Técnicas de análisis de datos con SPSS Madrid: Prentice Hall, 2009. ISBN: 9788483226018

Petersen J, Holmich, P. Evidence based prevention of hamstring injuries in sport. Br J Sports Med, 2005; 39(6), 319-323. http://doi.org/10.1136/bjsm.2005.018549

Pluim, B. M., Fuller, C. W., Batt, M. E., Chase, L., Hainline, B., Miller, S., ... \& Wood, T. O. (2009). Consensus statement on epidemiological studies of medical conditions in tennis, April 2009. Br J Sports Med, 43(12), 893-897. https://doi.org/10.1097/JSM.0b013e3181be35e5

Rae K, Orchard J. The Orchard Sports Injury Classification System (OSICS) version 10. Clin J Sport Med, 2007; 17(3), 201-204. https://doi.org/10.1097/JSM.0b013e318059b536

Rossi J, Vigouroux L, Barla C, Berton E. Potential effects of racket grip size on lateral epicondilalgy risks. Scand J Med Sci Sports, 2014; 24(6), 462-470. https://doi.org/10.1111/sms.12204 
Schoffl V, Morrison A, Hefti U, Ullrich S, Kupper T. The UIAA Medical Commission injury classification for mountaineering and climbing sports. Wilderness Environ Med, 2011; 22(1), 46-51. https://doi.org/10.1016/j.wem.2010.11.008

Steinbruck K. Epidemiology of sports injuries--25-year-analysis of sports orthopedic-traumatologic ambulatory care. Sportverletz Sportschaden, 1999; 13(2), 38-52. https://doi.org/10.1055/s-2007-993313

Thacker SB, Gilchrist J, Stroup DF, Kimsey CD, The impact of stretching on sports injury risk: a systematic review of the literature. Med Sci Sports Exerc, 2004; 36(3), 371-378. https://doi.org/10.1249/01.mss.0000117134.83018.f7

Thacker SB, Stroup DF, Branche CM, Gilchrist J, Goodman RA, Porter Kelling E. Prevention of knee injuries in sports. A systematic review of the literature. J Sports Med Phys Fitness, 2003; 43(2), 165-179. PMID:12853898

Winge S, Jørgensen U, Lassen NA. Epidemiology of injuries in Danish championship tennis. Int J Sports Med, 1989; 10(5), 368-371. https://doi.org/10.1055/s-2007-1024930

WMA. World Medical Association. Declaration of Helsinki: ethical principles for medical research involving human subjects. JAMA. 2013;310(20):21912194. https://doi.org/10.1001/jama.2013.281053

Número de citas totales / Total references: 32 (100\%)

Número de citas propias de la revista / Journal's own references: $0(0 \%)$

Rev.int.med.cienc.act.fís.deporte - vol. 19 - número 76 - ISSN: 1577-0354 